CLAWAR2016: 19th International Conference on

Climbing and Walking Robots and the Support

Technologies for Mobile Machines, London, UK, 12-14

September 2016, World Scientific Publishing Company, Singapore, ISBN: 978-981-3149-12-0.

\title{
STIFFNESS CONTROL OF BIONIC JOINT FOR A MUSCULOSKELETAL LEG MECHANISM*
}

\author{
JINGTAO LEI \\ School of Mechatronic Engineering and Automation, Shanghai University, Shanghai, \\ 200072, China \\ M. O. TOKHI \\ Department of Automatic Control and Systems Engineering, The University of Sheffield, \\ Sheffield, UK \\ JIANMIN ZHU \\ College of Mechanical Engineering, University of Shanghai for Science and Technology, \\ Shanghai, 200093, China

\begin{abstract}
Pneumatic artificial muscles (PAMs) have properties similar to a biological muscle, and are widely used in robotics. A musculoskeletal leg mechanism driven by PAMs is presented in this paper for a quadruped robot to achieve jumping movement with relatively higher-speed. There is contact force between foot and environment of robot with high-speed. In order to reduce the impact and improve the movement performance, the joint stiffness is controlled in real-time with contact stiffness and motion speed so as to reduce the impact due to contact force between the robot foot and the environment at high-speeds and improve the movement performance. A synchronous control method for position and stiffness is used. PID and BP neural network controls are developed for the antagonistic knee joint. Experimental investigations, providing a comparative assessment of performance of the PID and neural network approaches are carried out. A close match is achieved between theoretical analysis and experimental results.
\end{abstract}

\section{Introduction}

The development of bionic robots take inspiration from natural biological principles so as to achieve improved robot performance in terms of skeletal muscle coordination, smart structure, movement style, joint variable stiffness etc. [1]. As pneumatic artificial muscles (PAMs) have many desirable characteristics,

\footnotetext{
" This work is supported by National Natural Science Foundation of China Grant Number 51375289, National Natural Science Foundation of Shanghai Grant Number 13ZR1415500.
} 
such as flexibility similar to biological muscles, high power to weight ratio, high power to volume ratio, and inherent compliance they are widely used in various robotic systems [2]. In recent years, legged robots are developed using PAM as driver instead of electric motor to achieve desired high-speed movement of the legged robots [3, 4].

Quadruped robots have high load performance, are stable and have simpler structure as compared to six-legged or eight-legged robots. Several quadruped robots have been developed, and these are mostly driven by electrical motor or hydraulic actuators, with movement styles ranging from static walking to dynamic jumping and running [5-8]. The dynamic motion, such as jumping or running of a quadruped robot is characterised by large instantaneous forces and short durations. As PAM allows dynamic and agile movements for the robot with the property of light-weight and large amount of energy converted in short period of motion, some legged robots with musculoskeletal leg mechanism for high-speed movement have been developed recently using PAMs.

When a quadruped robot moves with high-speed, there is a momentary contact force between the robot foot and the environment. Thus, the key problem to solve is how to effectively reduce the impact so as to achieve soft landing of the robot during jumping. To reduce the non-continuous contact force between the foot and the environment, and for the robot to interact with the environment in a flexible manner, a suitable control method is needed. This will require controlling both the joint trajectory and joint stiffness so as to allow changes in real-time dynamic contact stiffness and load, and lead to good motion performance with high speed.

Tsujita et al. [9] have developed PAMs driven biped robot and joint stiffness analysis in walking. They have studied the relationship between muscle tone and locomotion performance. Nakamura et al. [10] have designed an antagonistic bionic joint driven by PAMs. They have adopted mechanical equilibrium model-based proportional-integral (PI) control method and have carried out experimental study on the positon and stiffness decoupling control. They have controlled the stiffness with a fixed value, and their joint position control accuracy is relatively low. Wang et al. [11] have developed a quadruped robot driven by PAMs. They have adopted a basic position control method and model-based position control method to control the joint positon. Their experimental investigations show variation of joint stiffness with time. Kang [12] has presented an antagonistic bionic joint driven by PAMs and has studied the force control method. The experimental results show that the sliding mode control algorithm could achieve better force tracking. But the joint stiffness control has not been studied. 
A musculoskeletal leg mechanism driven by PAMs for quadruped robot is presented. The jumping process is divided into three phases. The dynamics of the bionic leg during one jumping cycle is derived. The stiffness of the antagonistic knee joint driven by two FESTO PAMs is then obtained. A synchronous control method for joint position and stiffness is developed with proportional-integral-derivative (PID) and backpropagation (BP) neural network $(\mathrm{NN})$, and experimental investigations are carried out to verify the accuracy of theoretical analysis and control algorithm.

\section{Mechanical Configuration}

According to the design principle that the robot should have simple mechanical structure and better bionic characteristic, the robot's leg mechanism is developed so as to replicate the muscles arrangement of a quadruped animal, and its ranges of joint displacement. The bionic robot leg is driven by four PAMs and has three active joints and one passive joint, namely side-swing hip joint, hip joint, knee joint and passive ankle joint. The side-swing hip joint is mainly used for spinning gait of the quadruped robot, it has a slow response, and is designed with one PAM and one spring. The hip joint is also driven by one PAM and one spring. The knee joint is designed as an antagonistic joint driven by two PAMs, as shown in Figure 1. The structural parameters of the antagonistic knee joint are shown in Figure 2.

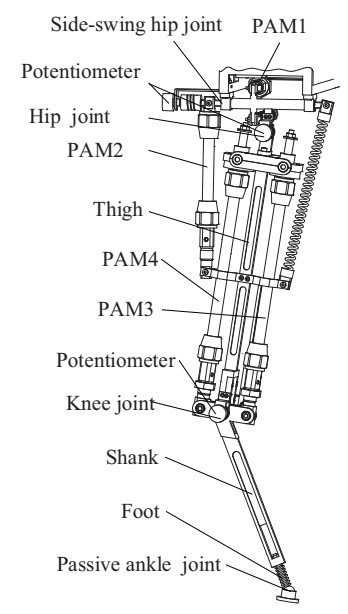

Figure 1. Musculoskeletal leg mechanism.

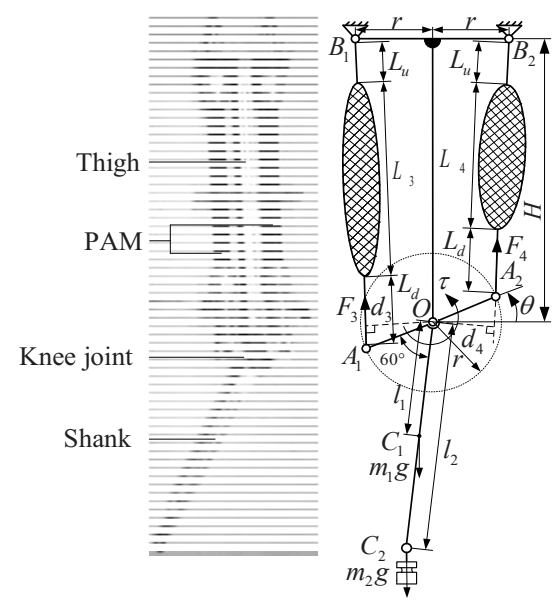

Figure 2. Antagonistic knee joint.

While the bionic leg jumps with high-speed, there is instant non-continuous 
impact force between the foot and the environment. Effective reduction of the contact force to achieve soft landing constitute the key to high-speed movement. In order to reduce the impact force between the foot and the environment, while the joint position is controlled, it is required to control the joint stiffness.

\section{Driving Torque and Stiffness Analysis of the Antagonistic Knee Joint}

\subsection{Driving Torque of the Knee Joint}

The driving torque of the knee joint can be expressed as:

$$
\boldsymbol{\tau}=\left(m_{1} l_{1}+m_{2} l_{2}\right) g \sin (\pi / 6-\theta)+\mathbf{F}_{4} d_{4}-\mathbf{F}_{3} d_{3}
$$

where, $\boldsymbol{\tau}$ is joint driving torque vector. $\theta$ is joint angle. $m_{1}, m_{2}$ are equivalent masses of the knee joint and external load, respectively. $l_{1}, l_{2}$ are distances from point $O$ to the equivalent mass centre $C_{1}$ and external load acting point $C_{2}$, respectively. $g$ is gravity acceleration. $\mathbf{F}_{3}$ and $\mathbf{F}_{4}$ are the output forces of PAM3 and PAM4. The PAM type used is MAS-10-180N-AA-MC-O from FESTO Company, whose axial output force was determined experimentally as:

$$
\mathbf{F}_{i}=\left(a_{1}+a_{2} p_{i}\right)+\left(b_{1}+b_{2} p_{i}\right) \varepsilon_{i}+\left(c_{1}+c_{2} p_{i}\right) e^{-0.3 \varepsilon_{i}}
$$

where, $a_{1}=-245.8, a_{2}=109, b_{1}=3.216, b_{2}=-3.442, c_{1}=255.6, c_{2}=-13.18$.

$p$ is the inner gas pressure of PAM. $\varepsilon$ is the contraction rate of PAM. $\varepsilon_{i}=1-L_{i}(t) / l_{0} \cdot L_{i}(t)$ is the real-time length of PAM. $l_{0}$ is the initial length of PAM, $l_{0}=180 \mathrm{~mm} . d_{3}, d_{4}$ are the force arms of $\mathbf{F}_{3}, \mathbf{F}_{4}$ about point $O$ respectively.

$$
\begin{gathered}
d_{3}=\frac{r(H \cos \theta+r \sin \theta)}{\sqrt{(H+r \sin \theta)^{2}+(r-r \cos \theta)^{2}}}, d_{4}=\frac{r(H \cos \theta-r \sin \theta)}{\sqrt{(H-r \sin \theta)^{2}+(r-r \cos \theta)^{2}}} \\
L_{3}(t)=\sqrt{(H+r \sin \theta)^{2}+(r-r \cos \theta)^{2}}-\left(L_{u}+L_{d}\right) \\
L_{4}(t)=\sqrt{(H-r \sin \theta)^{2}+(r-r \cos \theta)^{2}}-\left(L_{u}+L_{d}\right)
\end{gathered}
$$

where, $H, r$ are equivalent length and rotation radius of joint respectively; $H=370 \mathrm{~mm}, r=30 \mathrm{~mm}$.

\subsection{Stiffness of the Knee Joint}

Suitable joint stiffness is the key to achieving high-speed dynamic movement of the bionic leg. In order to obtain the desired joint stiffness for the bionic leg to 
achieve the desired jumping movement, the relationship between the joint stiffness and the gas pressure of PAMs needs be determined. The variation of joint stiffness of the musculoskeletal leg mechanism with the gas pressure of PAMs can be obtained from the dynamic model of the leg mechanism [11]. The joint stiffness can be derived from the joint driving torque as:

$$
K_{\theta}=-\frac{d \boldsymbol{\tau}}{d \theta}=-\frac{d\left(\left(m_{1} l_{1}+m_{2} l_{2}\right) g \sin (\pi / 6-\theta)+\mathbf{F}_{4} d_{4}-\mathbf{F}_{3} d_{3}\right)}{d \theta}
$$

where, $K_{\theta}$ is the knee joint stiffness. The negative sign indicates that the torque is in the opposite direction with the joint rotating.

Let $d_{3}=d_{4}=r$, then $K_{\theta}=\left(m_{1} l_{1}+m_{2} l_{2}\right) g \cos (\pi / 6-\theta)+r\left(\frac{d \mathbf{F}_{3}}{d \theta}-\frac{d \mathbf{F}_{4}}{d \theta}\right)$

where, $\quad \frac{d \mathbf{F}_{i}}{d \theta}=\frac{d \mathbf{F}_{i}}{d \varepsilon_{i}} \cdot \frac{d \varepsilon_{i}}{d \theta}=\left(\frac{0.3\left(c_{1}+c_{2} p_{i}\right) e^{-0.3 \varepsilon_{i}}-\left(b_{1}+b_{2} p_{i}\right)}{l_{0}}\right) \frac{d L_{i}}{d \theta}$

$$
\begin{aligned}
& K_{\theta}=\left(m_{1} l_{1}+m_{2} l_{2}\right) g \cos (\pi / 6-\theta)+ \\
& \frac{r^{2}(H \cos \theta+r \sin \theta)}{\sqrt{(H+r \sin \theta)^{2}+(r-r \cos \theta)^{2}}} \frac{0.3\left(c_{1}+c_{2} p_{3}\right) e^{-0.3 \varepsilon_{3}}-\left(b_{1}+b_{2} p_{3}\right)}{l_{0}}+ \\
& \frac{r^{2}(H \cos \theta-r \sin \theta)}{\sqrt{(H-r \sin \theta)^{2}+(r-r \cos \theta)^{2}}} \frac{0.3\left(c_{1}+c_{2} p_{4}\right) e^{-0.3 \varepsilon_{4}}-\left(b_{1}+b_{2} p_{4}\right)}{l_{0}}
\end{aligned}
$$

\section{Experimentation}

When the quadruped robot moves with high speed, there is a momentary contact force between the foot and the environment. The key control requirement is to effectively reduce the impact so as to achieve soft landing of the robot. To reduce the non-continuous contact force, for the robot to interact with the environment in a flexible manner, an effective control method needs to be devised. Figure 3 shows the control structure for simultaneous joint trajectory and stiffness control. 


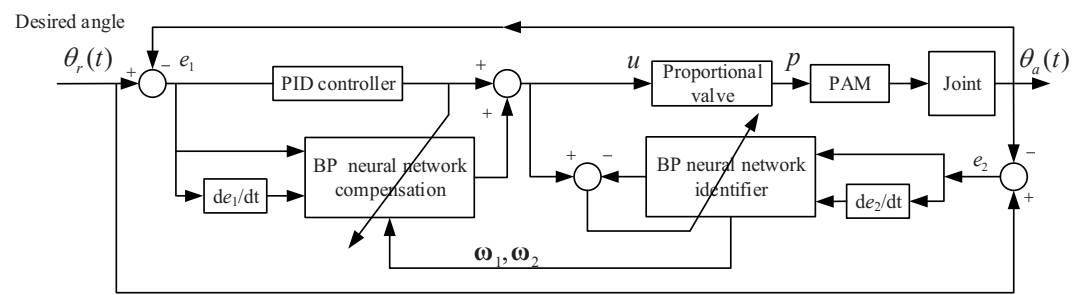

Figure 3. Control system structure of antagonistic knee joint.

The control system comprises traditional PID control and BP NN control for synchronised control of the position and stiffness of knee joint. The control system was implemented using the MATLAB Real-time Workshop. A sinusoidal joint displacement reference was set up, and experiments were carried out with constant and sinusoidal joint stiffness requirements. The experimental results are shown in Figures 4 and 5.

Figure 4 shows joint trajectory tracking with the desired stiffness represented as constant value or sinusoidal function. Figures 4(a) and 4(b) show the results using PID control during the constant stiffness and sinusoidal stiffness respectively. Figures 4(c) and 4(d) show the results using BP NN control during the constant stiffness and sinusoidal stiffness respectively.

Figure 5 shows the error in joint position and stiffness. Figures 5(a) and 5(b) show the angular displacement errors during the constant stiffness and sinusoidal stiffness respectively. Figures 5(c) and 5(d) show the stiffness errors during the constant stiffness and sinusoidal stiffness respectively. It is noted in the experimental results that: when the desired stiffness was constant, the joint position was relatively stable. In case of the desired stiffness given as a sinusoidal function, the joint angular displacement was volatile with changes in the joint stiffness. 

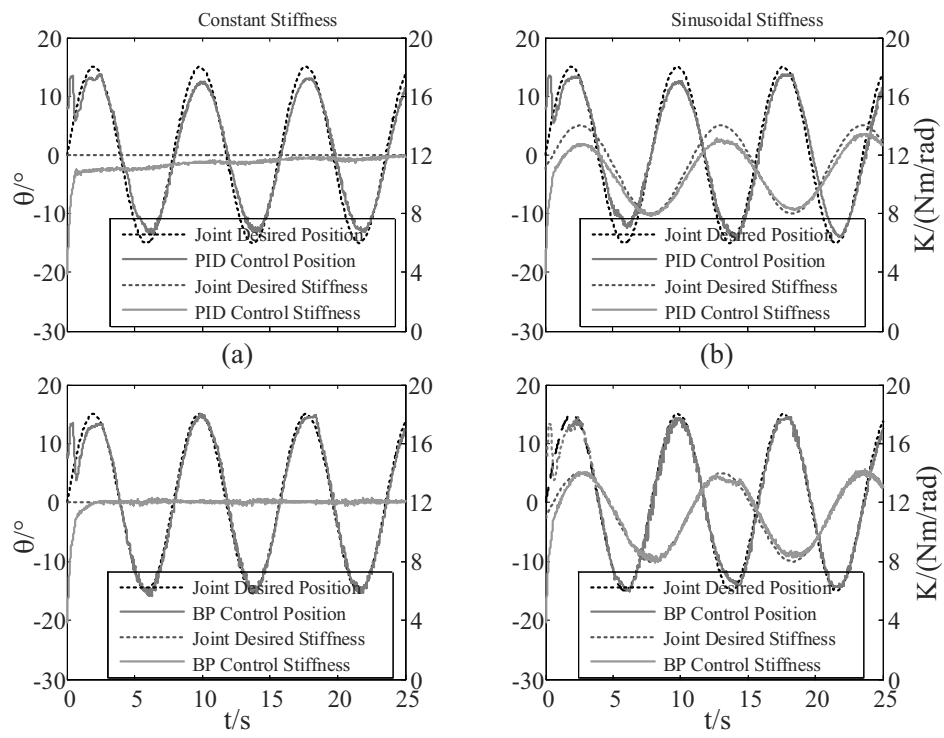

(d)

(e)

Figure 4. Experimental results of joint positon and stiffness control.

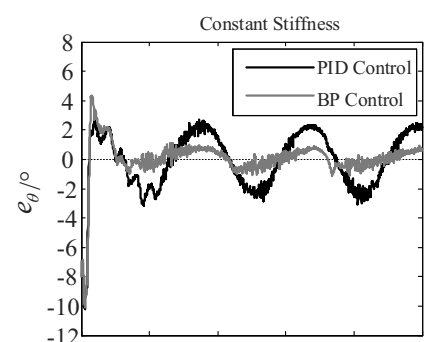

(a)

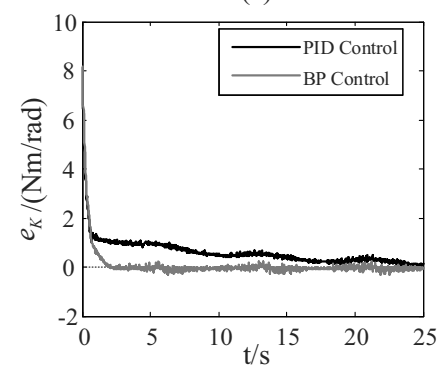

(c)

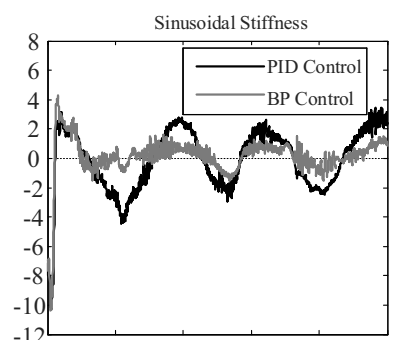

(b)

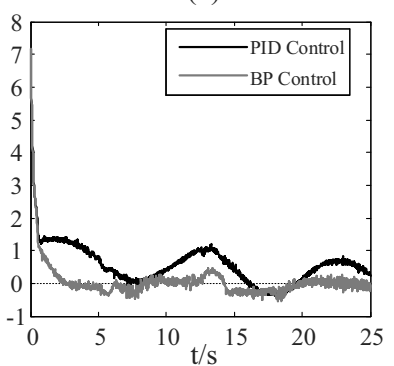

(d)

Figure 5. Errors in joint position and stiffness. 


\section{Conclusions}

A musculoskeletal leg mechanism driven by PAMs has been presented for a quadruped robot in jumping movement. The system dynamics have been derived to determine the relationship between the joint driving torque and the PAM gas pressure and further determining the joint stiffness. A PID and BP neural network control method has been developed for controlling the position and stiffness of the knee joint of the leg mechanism. The effectiveness of the control algorithm has been demonstrated through experimental investigations. Future work will focus on jumping planning strategies of trajectory and stiffness, and the position and stiffness control of the hip joint and knee joint according to the jumping movement planning strategy.

\section{References}

[1] A. J. Ijspeert. Biorobotics: Using robots to emulate and investigate agile locomotion. SCIENCE, 2014, Vol. 346, No. 6206:196-203.

[2] Georgios A., Georgios N., Stamatis M. A Survey on Applications of Pneumatic Artificial Muscles. 19th Mediterranean Conference on Control and Automation. Corfu, Greece, 2011:1439-1446.

[3] Niiyama R., Nishikawa S., Kuniyoshi Y. Biomechanical Approach to Open-Loop Bipedal Running with a Musculoskeletal Athlete Robot. Advanced Robotics, 2012, Vol. 26, No. 3-4:383-398.

[4] Nishikawa S., Yamada Y., Shida K. et al. Dynamic motions by a quadruped musculoskeletal robot with angle-dependent moment arms. International Workshop on Bio-Inspired Robots, Nantes, France, 2011.

[5] K. Narioka, A. Rosendo, A. Sproewitz. Development of a minimalistic pneumatic quadruped robot for fast locomotion. International Conference on Robotics and Biomimetics, Guangzhou, China, 2012: 307-311.

[6] X. Guo and Z. Pei. Study status and developing trend of hopping robots, Machinery Design and Manufacture, 2011, No. 11:173-175.

[7] Y. Yamada, S. Nishikawa, K. Shida. Neural-body coupling for emergent locomotion: A musculoskeletal quadruped robot with spinobulbar model. IEEE/RSJ International Conference on Intelligent Robots and Systems, San Francisco, CA, USA. 2011:1499-1506.

[8] Dong Jin Hyun, Sangok Seok, Jongwoo Lee et al. High speed trot-running: Implementation of a hierarchical controller using proprioceptive impedance control on the MIT Cheetah. International Journal of Robotics Research, 2014, Vol. 33, No. 11:1417-1445

[9] Tsujita K., Inoura T., Kobayashi T. et al. A Study on Locomotion Stability 
by Controlling Joint Stiffness of Biped Robot with Pneumatic Actuators. Motion and Vibration Control, 2009:305-314.

[10] Nakamura T., Tanaka D., Maeda H. Joint stiffness and position control of an artificial muscle manipulator for instantaneous loads using a mechanical equilibrium model. Advanced Robotics, 2011, Vol. 25, No. 3: 387-406.

[11] Wang X., Li M. T., Guo W. et al. Development of an Antagonistic Bionic Joint Controller for a Musculoskeletal Quadruped. IEEE/RSJ International Conference on Intelligent Robots and Systems, Tokyo, Japan, 2013:4466-4471.

[12] Bong Soo Kang. Compliance characteristic and force control of antagonistic actuation by pneumatic artificial muscles. Meccanica, 2014, Vol. 49, No. 3:565-574. 